\title{
System Analysis of LWDH Related Genes Based on Text Mining in Biological Networks
}

\author{
Mingzhi Liao, ${ }^{1}$ Yingbo Miao, ${ }^{2}$ Liangcai Zhang, ${ }^{3,4}$ Yang Wang, ${ }^{1}$ Rennan Feng, ${ }^{3,5}$ Lei Yang, ${ }^{3}$ \\ Shihua Zhang, ${ }^{6}$ Yongshuai Jiang, ${ }^{3}$ and Guiyou Liu ${ }^{3,7}$ \\ ${ }^{1}$ College of Life Sciences, Northwest A\&F University, Yangling, Shaanxi 712100, China \\ ${ }^{2}$ College of Pharmacy, Nankai University, Tianjin 300071, China \\ ${ }^{3}$ College of Bioinformatics Science and Technology, Harbin Medical University, Harbin 150081, China \\ ${ }^{4}$ Department of Statistics, Rice University, 6100 Main Street, Houston, TX 77005, USA \\ ${ }^{5}$ Department of Nutrition and Food Hygiene, School of Public Health, Harbin Medical University, Harbin 150081, China \\ ${ }^{6}$ College of Life Science, Anhui Agricultural University, Hefei 230036, China \\ ${ }^{7}$ Genome Analysis Laboratory, Tianjin Institute of Industrial Biotechnology, Chinese Academy of Sciences, Tianjin 300308, China
}

Correspondence should be addressed to Yongshuai Jiang; jiangyongshuai@gmail.com and Guiyou Liu; liuguiyou1981@163.com

Received 15 May 2014; Revised 2 July 2014; Accepted 3 July 2014; Published 27 August 2014

Academic Editor: Tatsuya Akutsu

Copyright (C) 2014 Mingzhi Liao et al. This is an open access article distributed under the Creative Commons Attribution License, which permits unrestricted use, distribution, and reproduction in any medium, provided the original work is properly cited.

\begin{abstract}
Liuwei-dihuang (LWDH) is widely used in traditional Chinese medicine (TCM), but its molecular mechanism about gene interactions is unclear. LWDH genes were extracted from the existing literatures based on text mining technology. To simulate the complex molecular interactions that occur in the whole body, protein-protein interaction networks (PPINs) were constructed and the topological properties of LWDH genes were analyzed. LWDH genes have higher centrality properties and may play important roles in the complex biological network environment. It was also found that the distances within LWDH genes are smaller than expected, which means that the communication of LWDH genes during the biological process is rapid and effectual. At last, a comprehensive network of LWDH genes, including the related drugs and regulatory pathways at both the transcriptional and posttranscriptional levels, was constructed and analyzed. The biological network analysis strategy used in this study may be helpful for the understanding of molecular mechanism of TCM.
\end{abstract}

\section{Introduction}

Traditional Chinese medicine (TCM) is one important component of the medical drug system, which can treat disease systematically and is effective at recuperating the balance of the whole body in patients using herbal formulas (Fang-Ji in Mandarin) [1]. TCM usually uses several medicinal herbs that have different roles. The ingredients of these herbs have been organized into certain formulas that may have potential complex interaction effects. Considering the different natural properties of herbs, including hot, warm, cool, and cold, the interactions among herbs will be difficult to be understood $[2,3]$.
In order to understand the complex effects of TCM formulas in the whole body, research about molecular targets of formula may be necessary. Some previous studies have found important target genes of TCM. Li et al. analyzed the target genes of TCM [4-6]. Based on the target prediction of TCM compounds, Liang et al. tried to construct the network about one case of TCM, which represents the newest progress of network approach in pharmacology [7]. Some researches tried to construct the relationship between TCM and disease in silico $[8,9]$. Fang et al. collected the target genes of TCM into a database TCMGeneDIT and analyzed those genes [10]. Furthermore, some researchers have generated a comprehensive database for TCM [11]. Based on the accumulation of 
TCM modernization, Chen group provided web servers for virtual screening and de novo drug design $[12,13]$. However, suitable methods for determining how the target genes work in the complex environment of the whole body have not been developed.

Complex biological systems can be broken down into interacting networks composed of nucleotides, RNA, proteins, drugs, foods, and so forth. Studies of biological networks are increasing the understanding of the mechanism of biological systems $[14,15]$. With the rapid development of computational systems biology, especially network biology, the mechanisms study about how TCM target genes work has changed from "one drug, one target" to "multidrugs, multitargets, biological networks" [16-18]. Based on these concepts, some studies constructed the drug targets network with PPINs [19]. Li group made their efforts to analyze the topological properties of drug targets in PPINs [20, 21]. Yang et al. also analyzed the topological characteristic of toxin targets in PPINs [22]. Though this research model is still in its infancy, computational systems pharmacology has also been highlighted in the medical drug development fields $[23,24]$. Previous studies attempted to construct and analyze the networks about TCM $[4,25]$. Considering the usefulness of analyzing biological networks to determine the complex effects of TCM target genes globally, we performed experiments to explore the topological properties of TCM in biological networks. These experiments had been previously applied to study social networks in social sciences [26].

In this study, candidate target genes of "Liuwei-dihuang" (LWDH, also known as Rehmannia Six, Six-Ingredient Rehmannia, or Rokumigan, a famous TCM formula that may nourish the balance of kidney yin yang) were obtained from previous studies using natural language mining technology. Then, the topological properties of LWDH genes were analyzed in PPINs. Interestingly, the LWDH genes were found to have high intensive centrality in PPINs. This indicates that the LWDH genes may have more important roles in the biological process than other genes. We also found that the distances within LWDH genes were smaller than other genes, which indicates that the LWDH genes may respond rapidly and synergistically to the common biological stimulation. For further understanding the inner molecular mechanism, a comprehensive network of LWDH genes was constructed and some modules were found.

\section{Materials and Methods}

2.1. Extraction of LWDH Genes Using Text Mining Technology. Considering the complexity of LWDH components and their complex interactions in vivo, little is known about the target genes of LWDH. The complex components of LWDH are comprised with six herbs. But their genomes of the six herbs that are components of LWDH have not been sequenced. Thus, determining the target genes of LWDH was critical for understanding the molecular mechanisms of LWDH. The existing research regarding $\mathrm{LWDH}$ genes is scattered in different groups around the world, even though the total number of LWDH target genes is small. Currently, researchers normally choose candidate genes for study by extensive literature review. However, scientific literature is now growing rapidly along with the development of the life science. The database of PubMed biomedical literature has over 23 million citations for biomedical literature from MEDLINE, life science journals, and online books. Thus, it is impossible for a researcher of a specific area to read all the literatures in his field, not to mention the papers in the related field. In this study, we obtained candidate LWDH target genes for study using the text mining technology.

We obtained LWDH genes for study by mining and analyzing the biomedical literature in PubMed with natural language processing technology. Considering the composition of LWDH, we extracted LWDH genes according to the components. As there are six herbs of LWDH, so we first decomposed LWDH with several search terms correspondingly, including Liu-wei-di-huang (LWDH, also known as Rehmannia Six, Six-Ingredient Rehmannia, or Rokumigan), Shan-zhu-yu (Fructus Corni), Ze-xie (Rhizoma Alismatis), Dan-pi (Cortex Moutan), Di-huang (Radix Rehmaniae), Fuling (Poria Cocos), and Shan-yao (Rhizoma Dioscoreae), and combined these search terms with "or" or "and" logic operators. Second, we extracted literature about Homo sapiens with the search terms in PubMed using eSearch and eFetch with the SQL statement. Third, we analyzed the literature with MEDLINE format files to get the gene name using the gene/protein names recognition software AbGene and filed the results manually [27]. At last, all the data were stored in the MySQL 5.0.90 database and reorganized into one list.

2.2. Protein-Protein Interaction Network Construction. The PPINs were constructed with a large number of proteins and their interactions, where the nodes denote proteins and the edges denote interactions between different nodes. PPINs are the most frequently used biological networks for research in computational system biology. PPINs are undirected networks that can be detected using multiple methods, including high-throughput and low-throughput experiments, such as yeast two-hybrid, affinity capture-MS, synthetic lethality, and reconstituted complex. In order to illustrate the robustness of our results to the primary dataset, we generated two different original datasets. The first is BioGrid and the other is HPRD $[28,29]$. Considering the false position rate, the highthroughput data for both datasets were filed out. In this study, the self-interactions were also eliminated.

2.3. The Topological Analysis of PPINs. To explore the roles that LWDH genes play in PPINs, this study focused on the centrality analysis of nodes. AS LWDH has the therapeutic effects of recovering the balance of kidney yin and yang in the complex whole-body environment, we hypothesized that the LWDH genes products in PPINs have different roles from other normal proteins. The centrality of one node in network has different measurements, including degree, betweenness, and clustering coefficient (Table 1) [30, 31]. We obtained as many centrality measurements as possible to explore the properties of LWDH genes. We obtained a total of 9 centrality measurements. Among the 9 measurements, 
TABle 1: Topological definition used in the study.

\begin{tabular}{|c|c|c|}
\hline Name & Definition & Description \\
\hline ASP & $\frac{\sum_{j \in N} d_{i j}}{|N||N-1|}$ & $\begin{array}{l}N \text { denotes the set of all nodes in the PPI network; } d_{i j} \text { denotes the } \\
\text { shortest path between node } i \text { and node } j\end{array}$ \\
\hline Betweenness & $B_{i}=\sum_{k \neq j \neq i \in V} \frac{\left|\sigma_{k i j}\right|}{\left|\sigma_{k j}\right|}$ & $\begin{array}{l}B_{i} \text { represents the betweenness of node } I, \sigma_{k j} \text { denotes shortest paths } \\
\text { between node pairs } k \text { and } j \text {, and } \sigma_{k i j} \text { denotes that pass through the } \\
\text { node } i\end{array}$ \\
\hline Closeness & $\frac{1}{\operatorname{avg}(L(n, m))}$ & $\begin{array}{l}L(n, m) \text { is the length of the shortest path between two nodes } n \text { and } m \text {. } \\
\text { The closeness centrality of each node is a number between } 0 \text { and } 1\end{array}$ \\
\hline Clustering coefficient & $\frac{2 n_{i}}{\left(k_{i}\left(k_{i}-1\right)\right)}$ & $n_{i}$ is the number of links between all neighbors of node $i$ \\
\hline Degree & $k_{i}$ & The number of links to node $i$ \\
\hline Eccentricity & $E$ & $\begin{array}{l}\text { The maximum node eccentricity }(E) \text { can be described as the network } \\
\text { diameter, which is the largest distance between two nodes }\end{array}$ \\
\hline Radiality & $R=D-\mathrm{ASPL}+1$ & $\begin{array}{l}\text { This attribute is a node centrality index computed by the diameter } \\
(D) \text { of a node } n \text { 's the connected component plus } 1 \text { and subtracting the } \\
\text { average shortest path length (ASPL) }\end{array}$ \\
\hline Stress & $p_{i}$ & $p_{i}$ is the number of shortest paths passing through $i$ \\
\hline $\begin{array}{l}\text { Topological } \\
\text { coefficient }\end{array}$ & $t_{i}=\frac{\operatorname{avg}(J(i, j))}{d_{i}}$ & $\begin{array}{l}t_{i} \text { represents the topological coefficient of node } I ; J(i, j) \text { is the number } \\
\text { of neighbors shared between the nodes } i \text { and } j \text {, plus one if there is a } \\
\text { direct link between } i \text { and } j \cdot \operatorname{avg}(J(i, j)) \text { is the average value of } J(i, j) . d_{i} \\
\text { is degree of node } i\end{array}$ \\
\hline
\end{tabular}

degree is the basic element in the topological analysis which was used to detect the number of edge links to the node. Besides, all other topological properties can be used to measure the centrality of nodes in biological networks. In general, the higher the centrality of one node, the more important roles it plays in biological networks. For detailed description, we took some properties as examples to illustrate their meanings. Average shortest path length (ASP) is defined as the average length between a node and all the nodes in biological networks. Closeness centrality is defined as the reciprocal of the average shortest path length of one node which can be used as a measure of how fast information spreads from a given node to all other reachable nodes in biological networks. In undirected biological networks (such as PPINs), clustering coefficient of a node is defined as the proportion of the observed connections between the neighbors of this node against the maximum number of possible connections among them. Clustering coefficient is used to indicate the close extent of the local neighborhood of one node. Topological coefficient is a relative measurement of the tendency of one node in biological networks to have shared interactive partners with other nodes. For more indepth interpretation of these concepts, one can get the exact definitions of these topological properties from Table 1.

In this study, we also paid close attention to the contacts within LWDH genes. There is one hypothesis that if the LWDH genes studies here had the same functions and acted on the same biological processes, it is expected that the distances among LWDH genes should be shorter than the average distance in background network. A shorter distance between two nodes in network will be helpful for information communication. Thus, this study will analyze the shortest path length among the LWDH genes.
Both the centrality analysis and shortest path length comparison were performed using the software Cytoscape version 3.0.0 [32, 33].

2.4. Construction and Analysis of Comprehensive Network about LWDH Genes. An integrated biological network was constructed (Figure 1). Firstly, we got the drug targets and drug-drug interaction information from DrugBank [34]. Second, the transcriptional regulation information was obtained from Transfac [35]. Third, we also detected the effects of posttranscriptional regulation on LWDH genes with microRNAs (miRNAs) and the related targets of miRNAs were from Tarbase, which only collected the results of experimental verification [36].

\section{Results}

3.1. LWDH Genes. Based on the text mining in PubMed, we first got all the references about LWDH. There are 151816 entries, including 3228 references, 107 species, and 145099 genes; see Table S1 in Supplementary Material available online at http://dx.doi.org/10.1155/2014/484926. As we focused on the genes that play roles in human, we considered genes on other species as false positive results. After the filter of species, we got total of 107 manually collected entries from which we can get LWDH genes with different searching conditions, including 96 unique genes related to LWDH in 61 literatures (Table S2). From the results, we found that dihuang is the core component of LWDH and had been most thoroughly investigated. Di-huang related genes were studied in 101 times. These studies included 93 unique genes in 56 


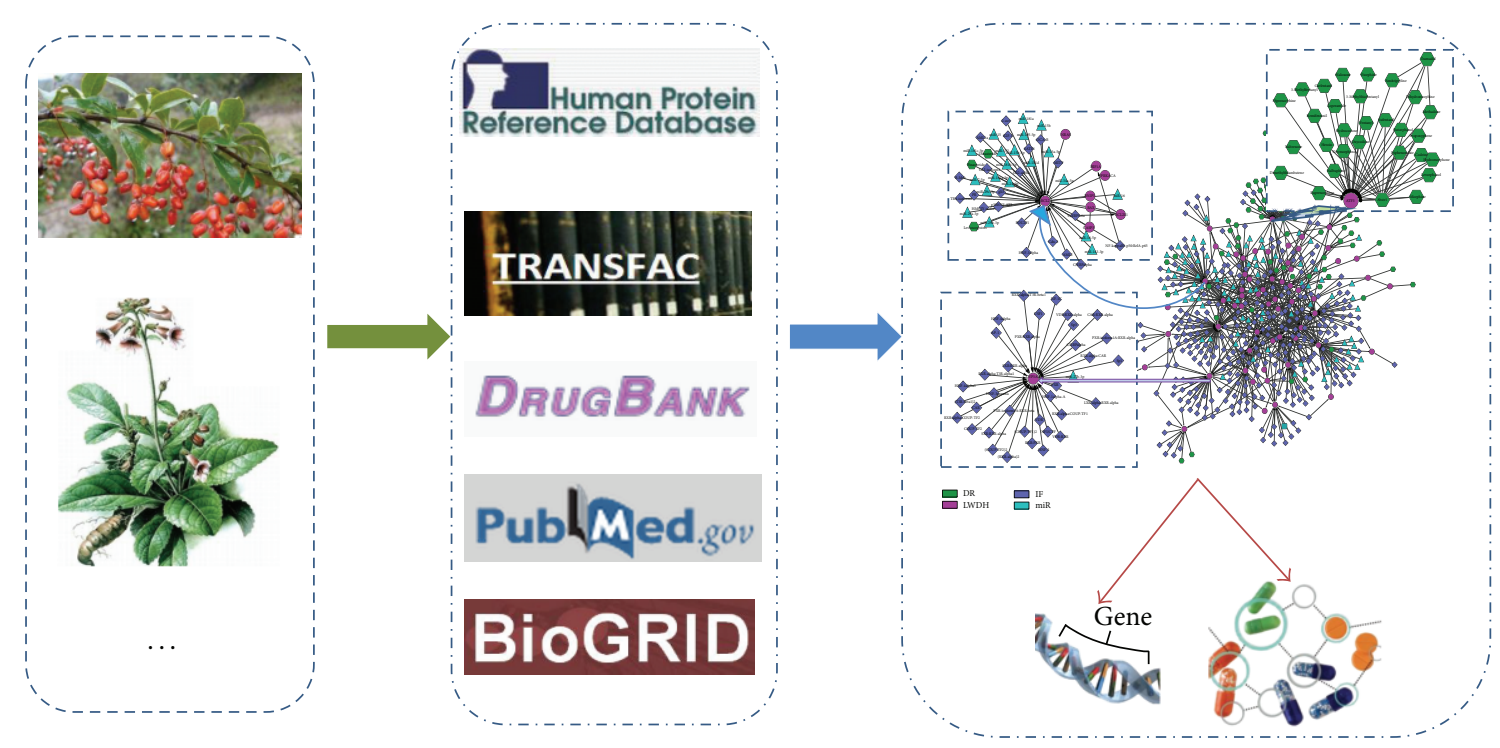

FIGURE 1: Workflow diagram of our approach to construct comprehensive biological network about LWDH genes. The left frame represents the component of LWDH. After the mining of related genes of LWDH based on natural language technology in PubMed, comprehensive information was obtained, including protein-protein interaction, transcription regulation, drug-drug interaction, and drug-target interaction (middle frame). At last, the comprehensive network of LWDH genes was constructed (right frame).

literatures. On the other hand, no research was found for zexie related genes. When the LWDH genes were mapped to the most component in PPINs, the total numbers of LWDH genes remain 71 and 65 in BioGrid and HPRD, respectively.

To verify the function of the LWDH genes, we performed a function enrichment analysis using gene ontology (Table 2). From the results, we found that the regulation of cell apoptosis and death is the main function of LWDH genes, which is consistent with previous study [37].

3.2. Protein-Protein Interaction Networks. There were a total of 10,996 nodes and 54,433 edges in BioGrid primary. After removing the isolated nodes, there were 10,768 nodes and 52,993 edges. Similarly, there were a total of 9,453 nodes and 36,888 edges in HPRD primary, and after removing the isolated nodes, there were 9,205 nodes and 36,748 edges. The LWDH genes that were mapped to the PPINs formed subnetworks that showed the core genes and their information communication path between them. Figure 2 shows that ESR1, STAT3, EP300, and so forthhave important positions in two subnetworks from both the BioGrid and HPRD datasets, respectively. The results indicate that these genes have important functions in the LWDH interaction environment.

3.3. Centrality of LWDH Genes in PPINs. In the centrality analysis of PPINs, 9 measurements in Table 1 were compared between the nodes which belong to LWDH genes (abbreviation as LWDH) and the nodes which do not belong to LWDH genes (abbreviation as Other). All the computations were processed with $t$-test in $R$ program. Results show that the LWDH genes have higher centrality than the other nodes in the PPINs (Tables 3 and 4). The BioGrid results showed that 8 out of the 9 measurements of the LWDH genes were significantly greater than the other nodes (Table 3 ). The HPRD dataset also supports the same tendency (Table 4).

3.4. Shortest Path Length Comparison. In order to explore the modularity within LWDH genes, we processed the computation about the shortest path length. As one famous herbal formula, LWDH is widely used and has been proved to be useful. If the LWDH gene is detected correctly, it is expected that the LWDH genes may show modularity and have closer connections inside themselves than the random chosen ones. In order to verify this hypothesis, the connection extent between each other among LWDH genes was compared with the background PPINs used shortest path length. As the expectation, Figure 3 supports that hypothesis. In the BioGrid datasets, the mean distance between the LWDH genes was 3.33, and if two nodes in the PPINs were chosen randomly, the expected distance was 4.20 (with $P<2.23 E-$ 308) (upper panel of Figure 3). Similar to the BioGrid results, the mean shortest path length of the LWDH genes in the HPRD was 3.28 , compared to 4.23 in the random situation (with $P=1.53 E-271$ ) (lower panel of Figure 3).

3.5. Comprehensive Network of LWDH Genes. The network reflects the impacts of both biological molecules and synthetic compounds (Figure 4). It contains 4 types of nodes, including $62 \mathrm{LWDH}$ genes, 301 transcriptional factors (TFs), 85 miRNAs, and 83 chemical compound drugs. Among these nodes, there were 5 types of edges, including 63 proteinprotein interactions (from both HPRD and BioGrid), $516 \mathrm{TF}$ transcriptional regulations, 113 miRNA posttranscriptional regulations, 106 drug-target relationships, and 40 drug-drug interactions (Table S3). From the network, we obtained 
TABLE 2: Functional enrichment analysis of LWDH genes in the biological process branch of GO.

\begin{tabular}{|c|c|c|c|c|c|}
\hline GOID & Term & Count & Count $\%$ & $P$ value & FDR \\
\hline GO:0043066 & Negative regulation of apoptosis & 18 & 18.9 & $4.00 E-12$ & $6.80 E-09$ \\
\hline GO:0043069 & Negative regulation of programmed cell death & 18 & 18.9 & $5.00 E-12$ & $8.50 E-09$ \\
\hline GO:0060548 & Negative regulation of cell death & 18 & 18.9 & $5.30 E-12$ & $8.90 E-09$ \\
\hline GO:0042981 & Regulation of apoptosis & 23 & 24.2 & $1.10 E-10$ & $1.90 E-07$ \\
\hline GO:0043067 & Regulation of programmed cell death & 23 & 24.2 & $1.40 E-10$ & $2.30 E-07$ \\
\hline GO:0010941 & Regulation of cell death & 23 & 24.2 & $1.50 E-10$ & $2.50 E-07$ \\
\hline GO:0010033 & Response to organic substance & 20 & 21.1 & $5.30 E-09$ & $9.00 E-06$ \\
\hline GO:0006916 & Antiapoptosis & 12 & 12.6 & $1.20 E-08$ & $2.10 E-05$ \\
\hline GO:0042127 & Regulation of cell proliferation & 20 & 21.1 & $2.20 E-08$ & $3.70 E-05$ \\
\hline GO:0009719 & Response to endogenous stimulus & 15 & 15.8 & $2.70 E-08$ & $4.70 E-05$ \\
\hline GO:0070482 & Response to oxygen levels & 10 & 10.5 & $6.20 E-08$ & $1.10 E-04$ \\
\hline GO:0009725 & Response to hormone stimulus & 14 & 14.7 & $6.90 E-08$ & $1.20 E-04$ \\
\hline GO:0043065 & Positive regulation of apoptosis & 14 & 14.7 & $4.30 E-07$ & $7.30 E-04$ \\
\hline GO:0043068 & Positive regulation of programmed cell death & 14 & 14.7 & $4.60 E-07$ & $7.90 E-04$ \\
\hline GO:0010942 & Positive regulation of cell death & 14 & 14.7 & $4.90 E-07$ & $8.30 E-04$ \\
\hline GO:0001666 & Response to hypoxia & 9 & 9.5 & $5.90 E-07$ & $1.00 E-03$ \\
\hline GO:0048545 & Response to steroid hormone stimulus & 10 & 10.5 & $8.60 E-07$ & $1.50 E-03$ \\
\hline GO:0001776 & Leukocyte homeostasis & 6 & 6.3 & $1.30 E-06$ & $2.20 E-03$ \\
\hline GO:0042592 & Homeostatic process & 17 & 17.9 & $1.90 E-06$ & $3.20 E-03$ \\
\hline GO:0006915 & Apoptosis & 15 & 15.8 & $3.30 E-06$ & $5.70 E-03$ \\
\hline GO:0051384 & Response to glucocorticoid stimulus & 7 & 7.4 & $3.80 E-06$ & $6.40 E-03$ \\
\hline GO:0012501 & Programmed cell death & 15 & 15.8 & $4.00 E-06$ & $6.70 E-03$ \\
\hline GO:0043029 & $\mathrm{T}$ cell homeostasis & 5 & 5.3 & $4.40 E-06$ & $7.40 E-03$ \\
\hline GO:0045884 & Regulation of survival gene product expression & 5 & 5.3 & $4.40 E-06$ & $7.40 E-03$ \\
\hline GO:0008219 & Cell death & 16 & 16.8 & $5.40 E-06$ & $9.10 E-03$ \\
\hline GO:0016265 & Death & 16 & 16.8 & $5.80 E-06$ & $9.90 E-03$ \\
\hline
\end{tabular}

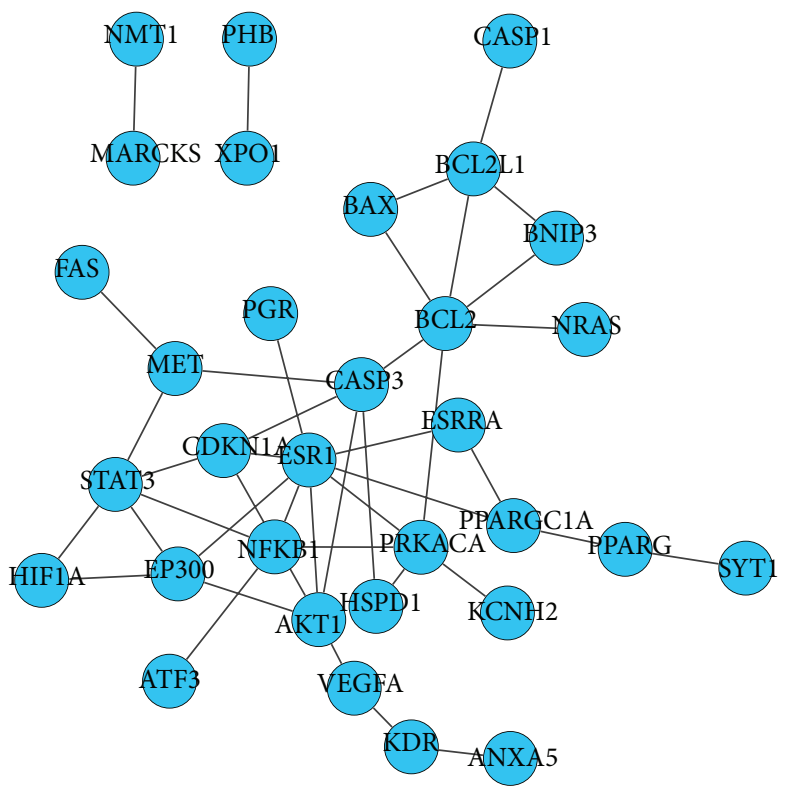

(a) HPRD

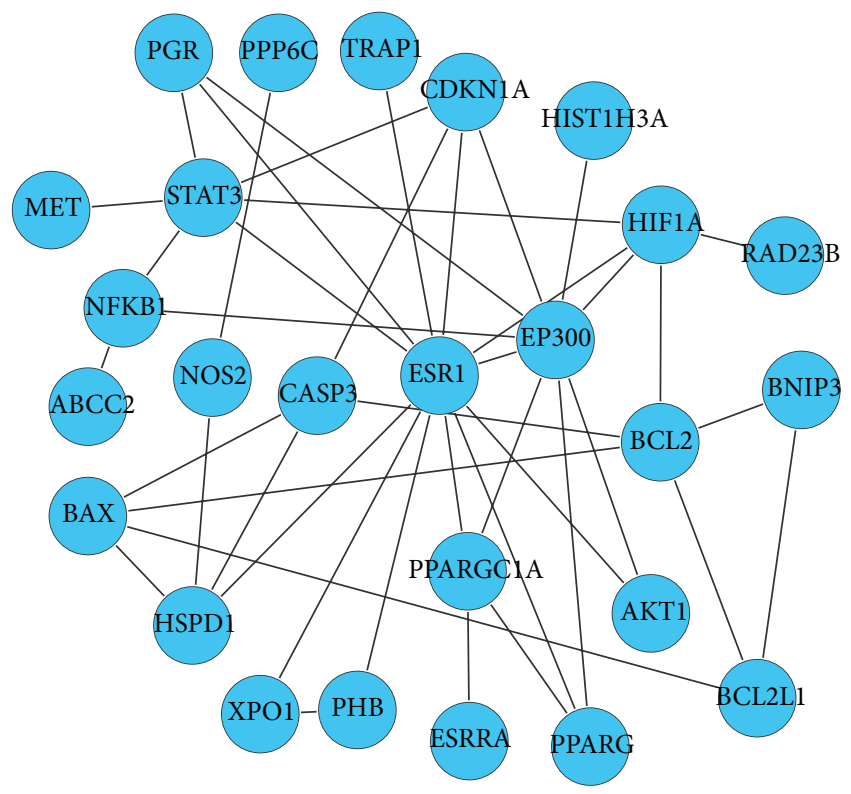

(b) BioGrid

FIgURE 2: Subnetwork of LWDH genes from BioGrid and HPRD. 
TABLE 3: Centrality analysis of LWDH genes in BioGrid.

\begin{tabular}{lcccc}
\hline Measurements & LWDH & Other & $P$ value & $-\log 10(P$ value $)$ \\
\hline ASP & 3.322458 & 3.756768 & $7.13 E-12$ & 11.14671 \\
Betweenness & 0.002336 & 0.000242 & $1.49 E-11$ & 10.82709 \\
Closeness & 0.308365 & 0.27118 & $7.01 E-18$ & 17.15445 \\
Clustering coefficient & 0.113448 & 0.156074 & 0.155779 & 0.807491 \\
Degree & 46.88732 & 9.596803 & $1.64 E-31$ & 30.78599 \\
Eccentricity & 7.309859 & 7.731794 & $2.9 E-08$ & 7.537242 \\
Radiality & 0.788867 & 0.749385 & $7.13 E-12$ & 11.14671 \\
Stress & 5075568 & 500694.1 & $1.36 E-16$ & 1 \\
Topological coefficient & 0.129467 & 0.178003 & 0.032223 & 1.491832 \\
\hline
\end{tabular}

TABLE 4: Centrality analysis of LWDH genes in HPRD.

\begin{tabular}{lcccc}
\hline Measurements & LWDH & Other & $P$ value & $-\log 10(P$ value $)$ \\
\hline ASP & 3.773832 & 4.229902 & $4.34 E-10$ & 9.362655 \\
Betweenness & 0.002235 & 0.000337 & $2.59 E-25$ & 24.58708 \\
Closeness & 0.270647 & 0.240556 & $3.86 E-15$ & 14.4135 \\
Clustering coefficient & 0.061171 & 0.10591 & 0.088168 & 1.054689 \\
Degree & 30.52308 & 7.82407 & $8.74 E-36$ & 35.05851 \\
Eccentricity & 9.323077 & 9.803829 & $9.55 E-08$ & 7.019855 \\
Radiality & 0.801869 & 0.769293 & $4.34 E-10$ & 9.362655 \\
Stress & 2551317 & 349608.7 & $1.47 E-29$ & 28.83402 \\
Topological coefficient & 0.103758 & 0.201088 & $3.1 E-05$ & 4.508613 \\
\hline
\end{tabular}
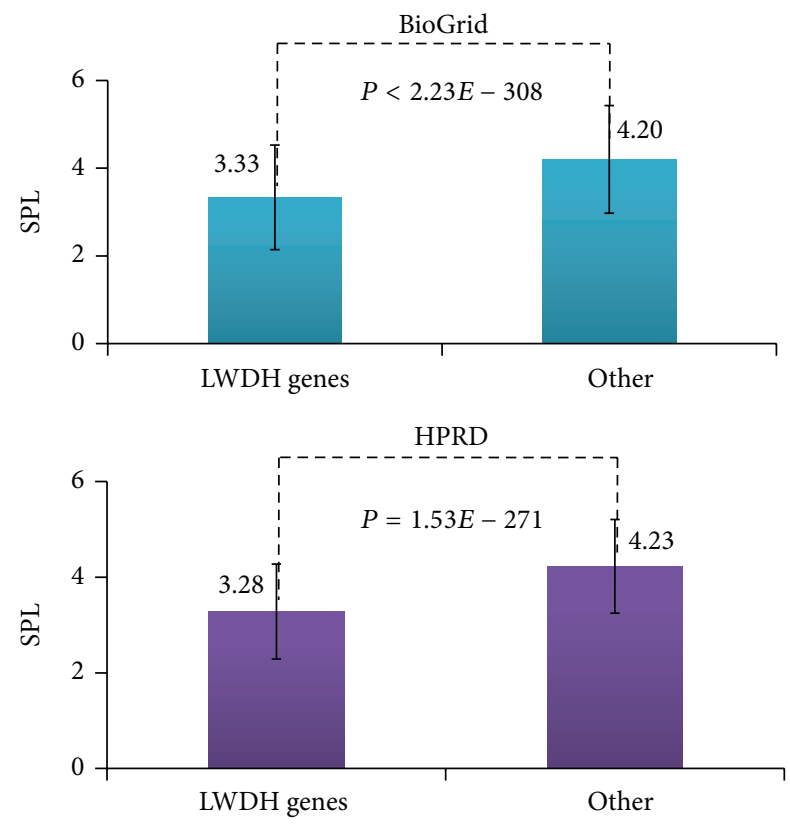

FIGURE 3: Comparison of the shortest path length between pairs of LWDH genes and pairs of other nodes. The left groups represent pairs of genes that are related to LWDH, and the right groups represent pairs of genes that are not related to LWDH. The measurement of SPL means the shortest path length of gene pairs in each group.

a panoramic view of the molecular interaction pathway of LWDH genes. Furthermore, the modules around the LWDH genes were found in the comprehensive networks. According to the types of nodes around the LWDH genes, the modules were classified into three types: the first one is drugs (drugmodule); the second one is TFs (TF-module); the third one is a mix of TFs and miRNAs (mixed-module). As a typical example of drug-module, ATF3 was targeted by a lot of drugs (upper right of Figure 4). Because ATF3 relates with both LWDH and lots of drugs, there should be common biological pathway through ATF3 that is shared by LWDH and these drugs. On the basis of common pathway about ATF3, this research may provide some novel molecular mechanism about the treating of disease with LWDH. CYP3A4 is one typical TF-module, which is targeted by lots of TFs (lower left of Figure 4). This result indicates that CYP3A4 may be a key crosstalk link to the pathways of these TFs in vivo. Considering the relationship between LWDH and these TFs through CYP3A4, we infer that LWDH implements its function of treating disease synergistically with these pathways of the TFs. As a TFs and miRNAs mixed-module, BCL2 is around with lots of TFs and miRNAs (upper left of Figure 4). This means that BCL2 may play roles from both transcriptional and posttranscriptional regulation levels.

\section{Discussion}

Facing the difficulty of studying the molecular mechanism about TCM targets, this research provided some novel approaches to reveal new characters of TCM genes in biological network. Through the example of $\mathrm{LWDH}$, we got their candidate target genes based on natural language text mining technology and found that they may play important 


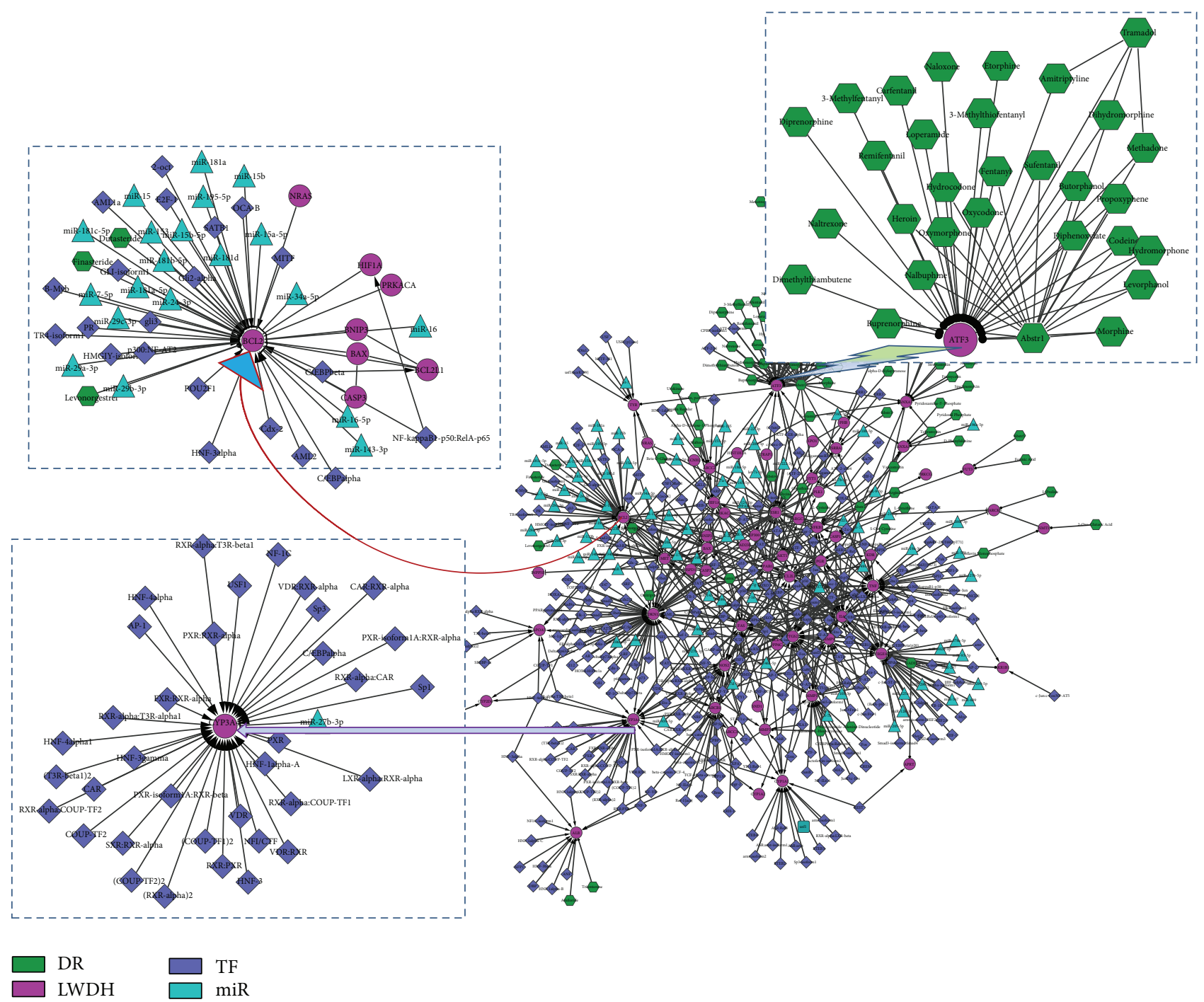

FIGURE 4: Comprehensive network of LWDH genes. Green represents drugs; purple represents LWDH genes; blue represents TFs; cyan represents miRNAs.

roles in PPINs. We also found that LWDH genes have relatively close communication in common biological process. Understanding the characteristics of TCM herbal formulas in complex biological networks, particularly through the molecular mechanism exploration, will not only benefit the modernization of TCM but may also be helpful for the development of new drugs $[2,17]$.

To address the lack of known target genes of LWDH, this study extracted all the known genes related to LWDH in Homo sapiens using natural program technology and deemed them LWDH genes. We used OMIM datasets as the criterion for validating the effectiveness of text mining methods for obtaining LWDH genes [38] (Table 5). The results showed that $\mathrm{LWDH}$ genes were mainly related to diseases of nosohemia and cancer, which is consistent with previous research $[7,39,40]$. Compared with Liang et al. research, our work provided all the genes in existing literatures and characterized the topological properties in biological networks firstly [7]. This strategy will be helpful for the traditional biological medicine research fields, which are still lacking genome information for the herbs that comprise LWDH. Using computational methods, this study obtained the LWDH genes from a large number of literatures rapidly. Considering the automation during the search of the literatures and the false positive results that were hidden in the literatures, the results of this study may need to be adjusted with new text searching technology and more abundant information in future.

Based on the extracted LWDH genes obtained from magnanimous literatures, we constructed two protein-protein interaction networks from different datasets and processed some topological analysis. Both analyses showed that the LWDH genes have higher centrality in PPINs, which indicate that they may play more important roles in complex wholebody environments. When one node has higher centrality properties in biological network, there would be more 
TABLE 5: Diseases related to LWDH genes in OMIM.

\begin{tabular}{lccl}
\hline Gene symbol & Location & MIM number & Disorders \\
\hline ALB & $4 \mathrm{q} 13.3$ & 103600 & $\begin{array}{l}\text { Analbuminemia; } \\
\text { dysalbuminemic } \\
\text { hyperthyroxinemia } \\
\text { APOA5 }\end{array}$ \\
BAX & $11 \mathrm{q} 23.3$ & 606368 & Hypertriglyceridemia \\
BCL2 & $19 \mathrm{q} 13.33$ & 600040 & Colorectal cancer \\
KDR & $18 \mathrm{q} 21.33$ & 151430 & Leukemia/lymphoma \\
MET & $4 \mathrm{q} 12$ & 191306 & Hemangioma, capillary infantile \\
PGR & $7 \mathrm{q} 31.2$ & 164860 & Renal cell carcinoma \\
PHB & $11 \mathrm{q} 22.1$ & 607311 & Progesterone resistance \\
\hline
\end{tabular}

information flow through it. These nodes may be regarded as key components in network, which help to maintain the stabilization of the molecular pathway. This result is consistent with the concepts of TCM function "balance" in regard to yin and yang, because only the proteins in the center of PPINs may be convenient to get the global information of body and can quickly respond to the wholebody environment $[2,41]$.

Interestingly, we also found that there is a smaller distance between the LWDH genes than would occur randomly. This indicates that the function of LWDH is focused on some particular biological process and that LWDH target genes have sufficient and quick information interflow. This is consistent with the well-known function of LWDH to help maintain and restore the balance of kidney yin yang [41]. These results also indicate that the LWDH herbal components are grouped together as the small distances between the nodes in the PPINs which mean the concentration of function. If one TCM herbal formula is expected to have good effects in treatment, its components may all focus on one main function and execute a common biological process synergistically.

Based on system information, a comprehensive network of LWDH genes was constructed. From that network, the effects of both biological molecules and artificial chemical drugs were clearly detected. Besides, some interesting results were found. Three types of modules around the LWDH genes were extracted from the comprehensive network. Genes in drug-modules are candidate targets of LWDH in treating disease. Observing genes in TF-modules can help researchers to detect the molecular mechanism of LWDH in vivo. Genes in mixed-modules may be helpful for exploring the epigenetic effects of LWDH in posttranscriptional regulation.

\section{Conclusions}

This research describes a new approach to explore the molecular mechanisms of TCM in complex biological networks. The topological properties observed may be helpful for the characterization and prediction of TCM target genes. The shortest path length comparison may also provide some criteria to estimate the rationality of TCM herbal formulas. Different modules in the comprehensive network of LWDH genes may provide a global perspective regarding TCM molecular mechanisms. With the development of computational system biology, their advantages in processing big data of biological medicine will be more significant.

\section{Conflict of Interests}

The authors declare that there is no conflict of interests regarding the publication of this study.

\section{Authors' Contribution}

Mingzhi Liao and Yingbo Miao contributed equally to this work.

\section{Acknowledgments}

This work was supported by the Fund of Northwest A\&F University; Shaanxi Provincial Natural Science Foundation (Grant no. 2014JQ3110); the National Natural Science Foundation of China (Grant nos. 31301938, 31200934, 81202184, and 81300945); the Construction Fund of Statistics Discipline in Anhui Agricultural University (XK2013031).

\section{References}

[1] S. Li, Z. Q. Zhang, L. J. Wu, X. G. Zhang, Y. D. Li, and Y. Y. Wang, "Understanding ZHENG in traditional Chinese medicine in the context of neuro-endocrine-immune network," IET Systems Biology, vol. 1, no. 1, pp. 51-60, 2007.

[2] J. Qiu, "China plans to modernize traditional medicine," Nature, vol. 446, no. 7136, pp. 590-591, 2007.

[3] F. Liang, L. Li, M. Wang et al., "Molecular network and chemical fragment-based characteristics of medicinal herbs with cold and hot properties from Chinese medicine," Journal of Ethnopharmacology, vol. 148, no. 3, pp. 770-779, 2013.

[4] S. Li, B. Zhang, D. Jiang, Y. Wei, and N. Zhang, "Herb network construction and co-module analysis for uncovering the combination rule of traditional Chinese herbal formulae," BMC Bioinformatics, vol. 11, supplement 11, article S6, 2010.

[5] S. Li, B. Zhang, and N. Zhang, "Network target for screening synergistic drug combinations with application to traditional Chinese medicine," BMC Systems Biology, vol. 5, supplement 1, article S10, 2011. 
[6] S. Zhao and S. Li, "Network-based relating pharmacological and genomic spaces for drug target identification," PLOS ONE, vol. 5, no. 7, Article ID el1764, 2010.

[7] X. Liang, H. Li, and S. Li, "A novel network pharmacology approach to analyse traditional herbal formulae: the Liu-WeiDi-Huang pill as a case study," Molecular BioSystems, vol. 10, no. 5, pp. 1014-1022, 2014.

[8] H.-C. Tang and C. Y.-C. Chen, "In silico design for adenosine monophosphate-activated protein kinase agonist from traditional chinese medicine for treatment of metabolic syndromes," Evidence-Based Complementary and Alternative Medicine, vol. 2014, Article ID 928589, 16 pages, 2014.

[9] H. J. Huang, Y. R. Jian, and C. Y. Chen, "Traditional Chinese medicine application in HIV: an in silico study," Journal of Biomolecular Structure and Dynamics, vol. 32, no. 1, pp. 1-12, 2014.

[10] Y.-C. Fang, H.-C. Huang, H.-H. Chen, and H.-F. Juan, "TCMGeneDIT: a database for associated traditional Chinese medicine, gene and disease information using text mining," BMC Complementary and Alternative Medicine, vol. 8, no. 1, article 58, 2008.

[11] C. Y.-C. Chen, “TCM Database Taiwan: the world's largest traditional Chinese medicine database for drug screening in silico," PLoS ONE, vol. 6, no. 1, Article ID e15939, 2011.

[12] K. Chang, T. Tsai, K. Chen et al., "iSMART: an integrated cloud computing web server for traditional Chinese medicine for online virtual screening, de novo evolution and drug design," Journal of Biomolecular Structure and Dynamics, vol. 29, no. 1, pp. 243-250, 2011.

[13] T. Tsai, K. Chang, and C. Y. Chen, "IScreen: World's first cloudcomputing web server for virtual screening and de novo drug design based on TCM database@Taiwan," Journal of ComputerAided Molecular Design, vol. 25, no. 6, pp. 525-531, 2011.

[14] W. Tao, X. Xu, X. Wang et al., "Network pharmacology-based prediction of the active ingredients and potential targets of Chinese herbal Radix Curcumae formula for application to cardiovascular disease," Journal of Ethnopharmacology, vol. 145, no. 1, pp. 1-10, 2013.

[15] W. Zhou and Y. Wang, "A network-based analysis of the types of coronary artery disease from traditional Chinese medicine perspective: potential for therapeutics and drug discovery," Journal of Ethnopharmacology, vol. 151, no. 1, pp. 66-77, 2014.

[16] C. T. Keith, A. A. Borisy, and B. R. Stockwell, "Multicomponent therapeutics for networked systems," Nature Reviews Drug Discovery, vol. 4, no. 1, pp. 71-78, 2005.

[17] A. L. Hopkins, "Network pharmacology: the next paradigm in drug discovery," Nature Chemical Biology, vol. 4, no. 11, pp. 682690, 2008.

[18] A. L. Hopkins, "Network pharmacology," Nature Biotechnology, vol. 25, no. 10, pp. 1110-1111, 2007.

[19] M. A. Yıldırım, K.-I. Goh, M. E. Cusick, A.-L. Barabási, and M. Vidal, "Drug-target network," Nature Biotechnology, vol. 25, no. 10, pp. 1119-1126, 2007.

[20] M. Zhu, L. Gao, X. Li et al., "The analysis of the drug-targets based on the topological properties in the human proteinprotein interaction network," Journal of Drug Targeting, vol. 17, no. 7, pp. 524-532, 2009.

[21] C. Wang, W. Jiang, W. Li et al., "Topological properties of the drug targets regulated by microRNA in human protein-protein interaction network," Journal of Drug Targeting, vol. 19, no. 5, pp. 354-364, 2011.
[22] L. Yang, W. Jizhe, W. Huiping, L. Yingli, Z. Yongchun, and W. Jiang, "Analysis and identification of toxin targets by topological properties in protein-protein interaction network," Journal of Theoretical Biology, vol. 349, pp. 82-91, 2014.

[23] M. Oshima, R. Torii, S. Tokuda, S. Yamada, and A. Koizumi, "Patient-specific modeling and multi-scale blood simulation for computational hemodynamic study on the human cerebrovascular system," Current Pharmaceutical Biotechnology, vol. 13, no. 11, pp. 2153-2165, 2012.

[24] T. Alan, "How can network-pharmacology contribute to antiepileptic drug development?" Molecular \& Cellular Epilepsy, vol. 1, no. 1, 2014.

[25] H.-P. Zhang, J.-B. Pan, C. Zhang, N. Ji, H. Wang, and Z.-L. Ji, "Network understanding of herb medicine via rapid identification of ingredient-target interactions," Scientific Reports, vol. 4, article 3719, 2014.

[26] S. Wasserman, Social Network Analysis: Methods and Applications, vol. 8, Cambridge University Press, Cambridge, Mass, USA, 1994.

[27] L. Tanabe and W. J. Wilbur, "Tagging gene and protein names in biomedical text," Bioinformatics, vol. 18, no. 8, pp. 1124-1132, 2002.

[28] A. Chatr-Aryamontri, B.-J. Breitkreutz, S. Heinicke et al., "The BioGRID interaction database: 2013 update," Nucleic Acids Research, vol. 41, no. D1, pp. D816-D823, 2013.

[29] T. S. Keshava Prasad, R. Goel, K. Kandasamy et al., "Human Protein Reference Database-2009 update," Nucleic Acids Research, vol. 37, supplement 1, pp. D767-D772, 2009.

[30] L. C. Freeman, "Centered graphs and the structure of ego networks," Mathematical Social Sciences, vol. 3, no. 3, pp. 291304, 1982.

[31] D. J. Watts and S. H. Strogatz, "Collective dynamics of "smallworld”" networks," Nature, vol. 393, no. 6684, pp. 440-442, 1998.

[32] R. Saito, M. E. Smoot, K. Ono et al., "A travel guide to Cytoscape plugins," Nature Methods, vol. 9, no. 11, pp. 1069-1076, 2012.

[33] Y. Assenov, F. Ramírez, S. E. Schelhorn, T. Lengauer, and M. Albrecht, "Computing topological parameters of biological networks," Bioinformatics, vol. 24, no. 2, pp. 282-284, 2008.

[34] C. Knox, V. Law, T. Jewison et al., "DrugBank 3.0: a comprehensive resource for "Omics" research on drugs," Nucleic Acids Research, vol. 39, supplement 1, pp. D1035-D1041, 2011.

[35] V. Matys, O. V. Kel-Margoulis, E. Fricke et al., “TRANSFAC and its module TRANSCompel: transcriptional gene regulation in eukaryotes," Nucleic Acids Research, vol. 34, supplement 1, pp. D108-110, 2006.

[36] T. Vergoulis, I. S. Vlachos, P. Alexiou et al., “TarBase 6.0: capturing the exponential growth of miRNA targets with experimental support," Nucleic Acids Research, vol. 40, no. 1, pp. D222-D229, 2012.

[37] Y. T. Tseng, F. R. Chang, and Y. C. Lo, “The Chinese herbal formula Liuwei dihuang protects dopaminergic neurons against Parkinson's toxin through enhancing antioxidative defense and preventing apoptotic death," Phytomedicine, vol. 21, no. 5, pp. 724-733, 2014.

[38] A. Hamosh, A. F. Scott, J. S. Amberger, C. A. Bocchini, and V. A. McKusick, "Online Mendelian Inheritance in Man (OMIM), a knowledgebase of human genes and genetic disorders," Nucleic Acids Research, vol. 33, no. supplement 1, pp. D514-D517, 2005.

[39] P. Y. Ma, Z. Y. Fu, Y. L. Su et al., "Modification of physicochemical and medicinal characterization of Liuwei Dihuang particles by ultrafine grinding," Powder Technology, vol. 191, no. 1-2, pp. 194-199, 2009. 
[40] N. Zhang and X. Wang, "Determination of constituents of Liuwei Dihuang Wan migrating to blood," Traditional Chinese Drug Research And Clinical Pharmacology, vol. 15, no. 3, pp. 174176, 2003.

[41] Y. Liu, M. Cheng, L. Wang, N. Zhao, H. Xiao, and Z. Wang, "Functional analysis of cultured neural cells for evaluating cold/cool- and hot/warm-natured Chinese herbs," The American Journal of Chinese Medicine, vol. 36, no. 4, pp. 771-781, 2008. 

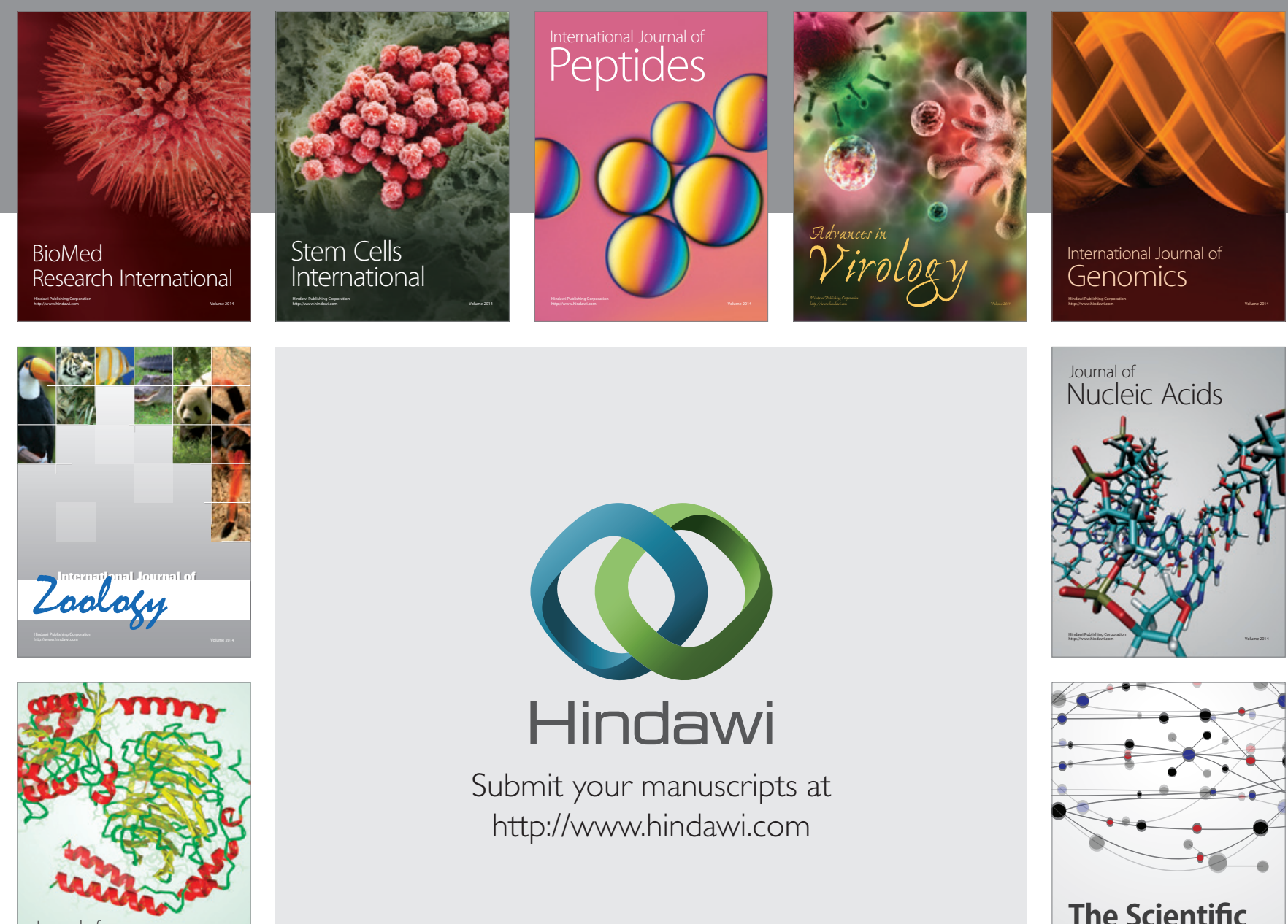

Submit your manuscripts at

http://www.hindawi.com

Journal of
Signal Transduction
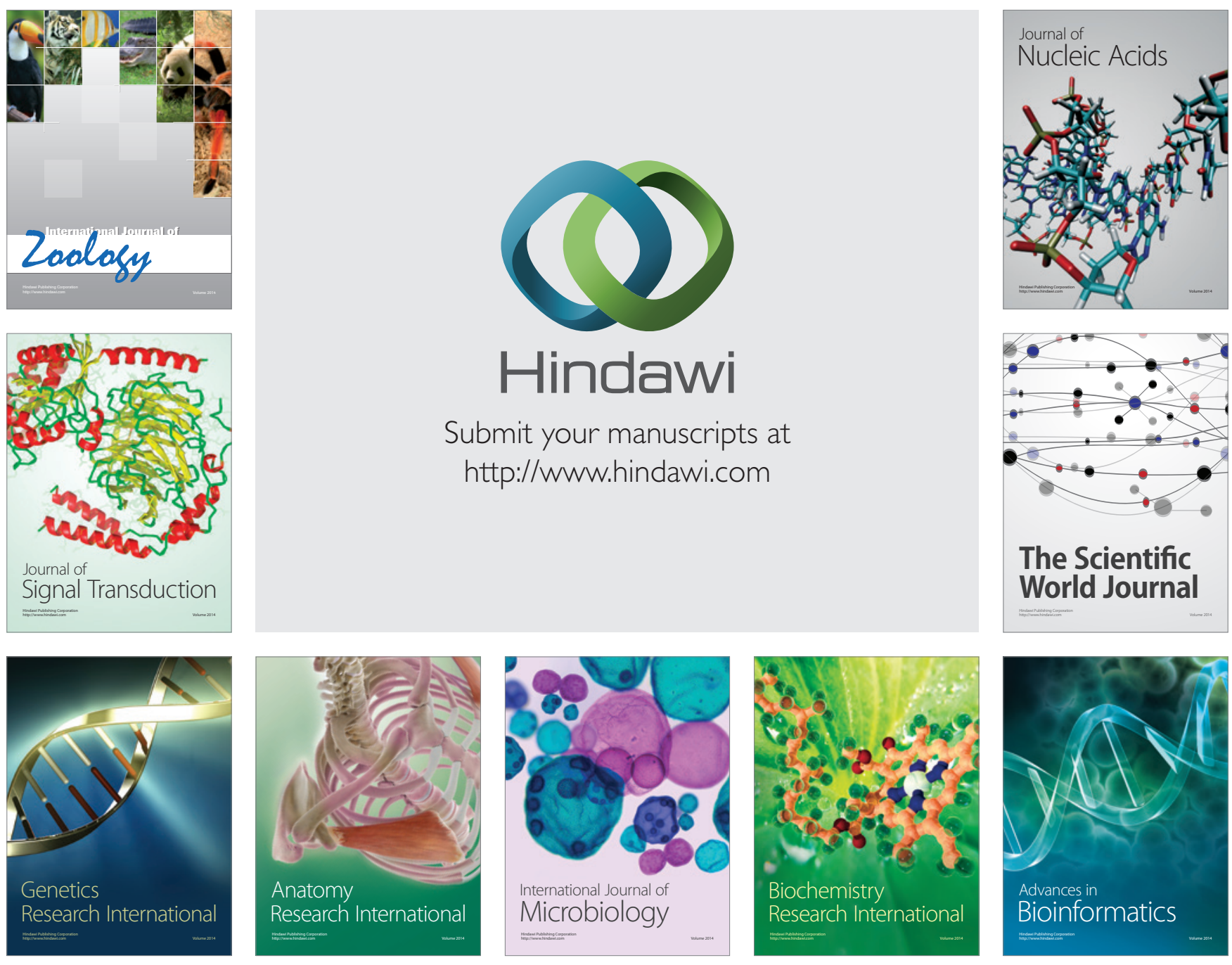

The Scientific World Journal
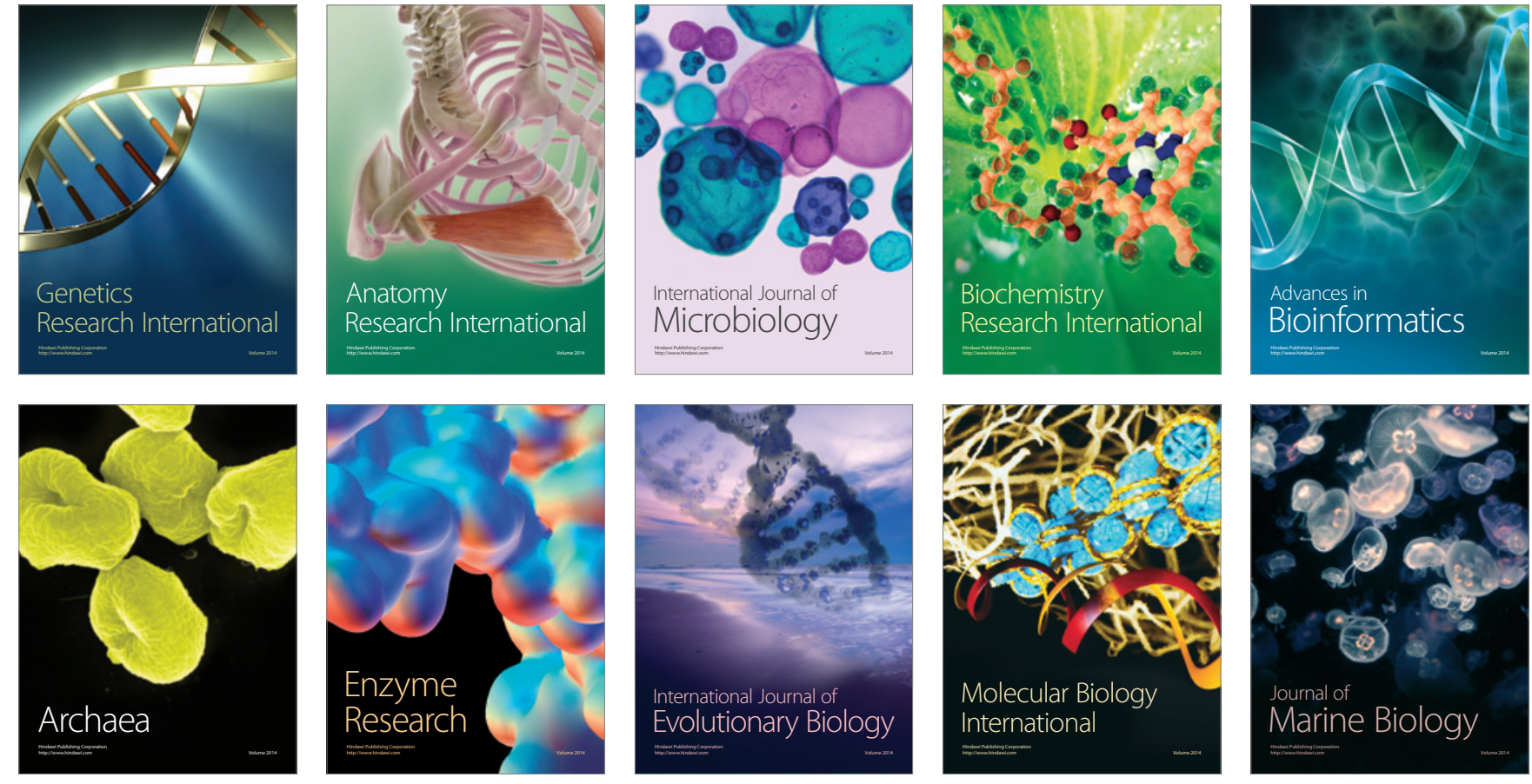